\title{
DATOS ETNOBOTANICOS DEL POBLADO DE HUAYLINGAS. CUENCA LA GALLEGA. MORROPON. PIURA
}

Aldo Ceroni Stuva ${ }^{1}$

\section{Resumen}

Se realizó un estudio etnobotánico en el poblado de Huaylingas (Morropón, Piura) con la finalidad de obtener información acerca del conocimiento sobre los recursos vegetales y técnicas autóctonas en un ecosistema de alta montaña. Se registraron actividades de subsistencia, técnicas de cultivo, de conservación de alimentos y una gran fuente de recursos fitogenéticos de uso potencial tanto en la alimentación como en la salud. Las 86 especies registradas fueron clasificadas en 8 categorías de uso, siendo 46 de ellas de uso medicinal.

Palabras clave: categorías de uso, etnobotánica, flujo de información, recursos fitogenéticos, técnicas autóctonas.

\begin{abstract}
An ethnobotanic study was conducted in the town of Huaylingas (Morropón, Piura) with the purpose of obtaining information on the current knowledge on plant resources and autoctonous techniques in a high mountain ecosystem. Subsistence activities, cultivation and food conservation techniques and a great source of phytogenetic resources with potential use as feedstuffs and in human health were registered. The 86 species registered were classified into 8 categories of use and 46 of them were of medical use.
\end{abstract}

Words key: Categories of use, ethnobotany, flow of information, phytogenetic resources, autoctonous techniques.

\section{Introducción}

Desde los comienzos de la humanidad, las plantas han ocupado un papel importante, sirviéndole al hombre como: alimento, en la construcción de sus casas, mobiliario, en la fabricación de telas, tintes, aceites, esencias, en instrumentos de caza, de guerra, como forraje, etc. (Infantes, 1962). El desconocimiento de la escritura en los antiguos pobladores del Perú no permitió dejar documento alguno que hubiera servido para conocer en forma directa y con exactitud el desarrollo de los acontecimientos. Sin embargo, los estudios etnobotánicos permiten rescatar una serie de conocimientos y técnicas ancestrales que los antiguos peruanos utilizaban con gran eficacia para el mejor aprovechamiento y conservación de los recursos naturales de su medio. La etnobotánica si bien es cierto que específicamente permite conocer la concepción autóctona, de la naturaleza y el mundo de las plantas de un determinado grupo étnico, también es cierto que es una de las mejores maneras de conocer el aprovechamiento de los recursos naturales, ya que todas las actividades de los seres humanos están principalmente relacionadas con las plantas. En cuanto a la sierra de Piura, hay en la actualidad algunos estudios etnobotánicos pero al parecer no son muy numerosos (Polia, 1989). El presente trabajo tuvo como objetivo realizar un estudio etnobotánico en el poblado de Huaylingas, para lo cual se registraron datos en cuanto a las actividades de subsistencia, técnicas de cultivo, técnicas de conservación de alimentos, así como del uso de las plantas.

\section{Materiales y método \\ Ubicación de la zona de estudio}

El Poblado de Huaylingas se ubica en la parte alta de la Cuenca La Gallega, en La Cordillera a 2700 msnm aproximadamente, en la Provincia de Morropón, Distrito de Santo Domingo, Departamento de Piura. Esta cuenca está ubicada al suroeste de la microregión andina central, dentro de la Gran Cuenca del Piura. La microregión andina central se ubica entre los $4^{\circ} 90^{\prime}$ y $5^{\circ} 10^{\prime} \mathrm{LS}$ y los $79^{\circ} 30^{\prime}$ y $80^{\circ} 10^{\prime}$ LO (Figura 1). La zona de estudio presenta un tipo de bosque húmedo siempre verde, con plantas como "higuerón", "suro", "paltón" y "puchuguero". En cuanto al clima, ésta presenta áreas húmedas con precipitaciones de 800 a $1000 \mathrm{~mm}$ por año.

Lugares de colecta

La información, así como el material botánico fue colectado en los alrededores: Florecer, Las Pircas y Cerro Negro

\section{Método}

En cuanto al procedimiento este consistió primero en establecer un flujo de información a través de la comunicación con los pobladores, entrevistas, etc. El flujo de información se obtuvo principalmente del Sr. Ángel Córdova Guerrero y de Manuel Jesús, su hijo

\footnotetext{
${ }^{1}$ Aldo Ceroni Stuva. Departamento Académico de Biología. Universidad Nacional Agraria La Molina. Av. La Universidad s/n. Apartado postal 456. Lima 100.Perú. Fax: 3496015. Dirección electrónica: aceroni $a$ lamolina edı ne.
} 
mayor. En segundo lugar se hizo una herborización de las especies útiles para todas y cada una de las actividades de la comunidad. La información obtenida fue ordenada y evaluada, mientras que las especies vegetales fueron determinadas en el Herbario Weberbauer de la Universidad Nacional Agraria La Molina (MOL) y en el Herbario del Museo de Historia Natural "Javier Prado", de la Universidad Nacional Mayor de San Marcos.

\section{Resultados y discusión}

Cómo comprenden su mundo vegetal

Huaylingas es un poblado que alberga aproximadamente 200 familias. Las plantas para ellos son sin lugar a dudas lo más importante por cuanto son la principal fuente de sus recursos tanto como alimento, material de construcción y medicina. Hay también una influencia de la religión en el sentido que previo a las siembras ellos invocan a Dios para tener éxito en sus cultivos y obtener buenas cosechas.

Las plantas las clasifican por su utilidad en: alimenticias, medicinales, para construcción, para leña, etc. Reconocen las plantas básicamente por el olor y el sabor, especialmente las hierbas medicinales.

En cuanto a la nominación de las partes de la planta usan los términos raíz, hojas, flores y frutos, a excepción de tallo, al que llaman "palo". Asimismo, llaman "tamo" a lo que queda de la cosecha y se usa como alimento para los animales.

Otros términos usados por ellos son: "monte" para los árboles no cultivados y "árbol" para los cultivados.

Actividades de subsistencia

La principal actividad de subsistencia es la agricultura. La ganadería es menos importante, ya que no tienen mucha agua como para preparar invernas. Las vacas comen hierbas naturales como Chusquea sp. "suro" y el llamado "tamo". En cuanto a las actividades agrícolas, tenemos que la siembra de tuberosas empieza en diciembre y la de granos en febrero, mientras que las cosechas las hacen desde mayo y agosto, para las tuberosas y granos, respectivamente. En noviembre, empieza la preparación de la tierra para tubérculos como el "olluco" y la "papa".

Entre las plantas que cultivan están: "maíz", "trigo", "cebada", "arveja", "papa", "olluco", "oca", "zanahoria", "ajo", "cebolla" y "zambumba". En el caso de la "papa" cultivan las variedades: Ranrainca, Nativa, Limeña, Yopata, Perrichola y Amapola.

Otra de las actividades que realizan es el trueque de productos que ellos producen por otros que no siembran. Por ejemplo: en los lugares de menor altitud no cultivan granos ni tubérculos, entonces cambian estos cultivos que si producen por "caña de Guayaquil" que no puede desarrollar en su sitio por el frío, pero que es muy necesario como material de construcción.
También realizan actividades como: recolección de leña, corte de árboles para obtener madera de construcción y recolección de semillas. Algunas plantas que usan para leña son: Baccharis latifolia "chilca" y Ocotea cernua "puchuguero"; para madera: Eucalyptus globulus "alcanfor", de donde obtienen 5 ó 6 tablones de cada tronco de $60 \mathrm{~cm}$. de diámetro aproximadamente. Cuando cortan estos últimos colectan semillas para preparar almácigos y obtener plantas para usarlas como cortinas rompevientos alrededor de sus cultivos.

La cría de sus animales, la obtención de algunos productos como: leche, manteca, etc. y la recolección de plantas medicinales, son las actividades de su vida cotidiana.

Técnicas autóctonas modificadas de cultivo

En el mes de noviembre empieza la preparación de la tierra para el cultivo de tubérculos. Para el caso de la "oca", abonan la tierra con estiércol de oveja y deshacen todos los terrones rompiéndolos con el "pilón", herramienta hecha de madera con una bola a un extremo (Foto 1). Las maderas que usan para elaborar esta herramienta pueden ser: Myrcia sp. "lanche", Oreocallis grandiflora "cucharillo" o Escallonia resinosa "chachacomo". Deshechos los terrones proceden a arar el terreno con bueyes que tiran el arado. Para el cultivo de la "papa", usan úrea cuando preparan la tierra.

Otras técnicas

Para la preparación de almácigos de "alcanfor" proceden de la siguiente manera: los frutos colectados con semillas son tendidos al sol por 3 días, luego sacan las semillas sacudiendo cada fruto. Preparan la tierra abonándola con estiércol de oveja; siembran las semillas y en 3 ó 4 meses obtienen los plantoncitos para el transplante.

Otra técnica observada entre los pobladores de este lugar fue el tratamiento de la madera cuando es cortada muy joven (verde): entierran los tablones por 3 meses aproximadamente antes de usarlos; de esta manera evitan que se raje.

Técnicas de conservación de alimentos

Los pobladores de Huaylingas usan algunas técnicas como el soberado o tabanque para conservar tubérculos. En este caso tienden la cosecha sobre un tabladillo hecho de Myrsine oligophylla "yuto": el denominado "tabanque". De esta manera, pueden conservar la cosecha hasta 3 ó 4 meses (Foto 2).

Para conservar granos guardan las cosechas en unos sacos de cuero de vaca bien cerrados: los denominados "zurrones". En cada saco se puede guardar hasta 13 almudas; cada almuda es aproximadamente 2 latas y cada lata tiene $12 \mathrm{~kg}$. Es decir, cada zurrón contiene hasta $310 \mathrm{Kg}$. De esta manera pueden conservar los granos 7 ó 10 años (Foto 3).

$\underline{\text { Usos de las plantas silvestres }}$

Además de la información recogida, se recolectó 
especies vegetales silvestres utilizadas por los pobladores, registrándose alrededor de 86 especies con usos variados, siendo 46 de uso medicinal. Una visita y entrevista al Sr. Gerardo Peña, curandero de Cerro Negro, permitió conocer el uso medicinal de muchas plantas. En general, se registraron hasta 8 categorías de uso: Alimenticias-Bebidas-Frutales; De carpintería y construcción; De higiene; Forrajeras; Medicinales; Para la buena suerte; Para leña y Para reforestar. En la tabla 1 se presenta una lista alfabética de los nombres vulgares y científicos de las plantas usadas en esta zona de estudio. Asimismo, en la tabla 2 se presentan las mismas ordenadas según su categoría de uso y en la tabla 3 las formas de uso.

\section{CONCLUSIONES}

1. Para los pobladores de Huaylingas, las plantas constituyen la principal fuente de sus recursos tanto como alimento, material de construcción y medicinales. Se registraron 86 especies útiles, de las cuales 46 tienen uso medicinal para 32 dolencias diferentes.

2. La clasificación de las plantas la hacen básicamente por su utilidad y el reconocimiento de las plantas medicinales principalmente por el olor y el sabor.

3. La nomenclatura usada para las partes de la planta son: raíz, palo (para el tallo), hojas, flores y frutos. Utilizando también las categorías etnobotánicas de "monte" para los árboles no cultivados y de "árbol" para los cultivados. El término "tamo" es utilizado para designar a los residuos de la cosecha que puede servir de pastura.

4. Las actividades de subsistencia en Huaylingas son: agricultura, intercambio de productos, recolección de leña y semillas, corte de árboles, crianza de animales, elaboración de productos $\mathrm{y}$ recolección de plantas medicinales.

5. Las actividades agrícolas empiezan en noviembre con la preparación del terreno para la siembra de tubérculos, que se cosechan en mayo. En febrero se siembran los cereales y se cosechan en agosto.

6. Las técnicas autóctonas son aplicadas: en el cultivo, utilizándose el arado tirado de buey y el pilón; en la preparación de almácigos y en el tratamiento de la madera cuando ha sido cortada algo verde.

7. Los alimentos son conservados utilizando técnicas como: El soberado o tabanque, para las tuberosas, y el de los zurrones, para los granos.

8. Se registraron 8 categorías de uso: AlimenticiasBebidas-Frutales, De carpintería y construcción, De higiene, Forrajeras, Medicinales, Para la buena suerte, Para leña y Para reforestar.

9. Huaylingas representa una buena fuente de recursos fitogenéticos que pueden ser potencializados, tanto en el campo de la salud como de la alimentación y que necesitarían un mayor estudio. Plantas como la "oca", el "olluco", el "pajul" y variedades de "papa"; o frutales como el "chicope" que se puede cultivar a altitudes mayores que la "papaya".

\section{AGRADECIMIENTOS}

En la realización del presente trabajo fue muy significativa la participación de la Bach. Alina Pace Guzmán y el apoyo de la Ing. Luz María Jiménez Castillo, Profesora del Instituto Superior Tecnológico "Santo Domingo de Guzmán", de Santo Domingo. La Profesora Jiménez estableció el contacto con el Sr. Angel Córdova Guerrero y nos acompañó a todos los lugares visitados durante el estudio. El autor también expresa su agradecimiento al Sr. Córdova, quien nos alojó en su casa y nos brindó toda la información requerida. Asimismo, un especial agradecimiento a la Dra. Emma Cerrate, Profesora de la Maestría en Botánica Tropical de la UNMSM por sus directivas y sugerencias en la investigación etnobotánica y a la Central Peruana de Servicios (CEPESER) de Piura por el apoyo financiero y logístico. Sin todos ellos hubiera sido difícil poder realizar el presente estudio.

\section{Literatura citada}

Alcalde M. 1990. Especies Agropastoriles para la zona altoandina. Arbolandino Pomata. Perú.

Bernex De Falen N. 1987. La Geografía Regional del Norte. Primera edición. Centro de Investigaciones y Promoción del Campesinado. (CIPCA)

Cerrate E. 1979. Plantas que curan las heridas del hombre y los animales. Primera parte. Boletín de Lima. $\mathrm{N}^{\circ} 3: 12-17$.

- 1980. Plantas que curan las heridas del hombre y los animales. Segunda parte. Boletín de Lima. $\mathrm{N}^{\circ} 4$. : 14-18.

CIGA. 1990. Cuaderno de Geografía Aplicada. Proyecto de Desarrollo Rural Integral de la Sierra Central del Departamento de Piura. PUCORSTOM. Primera parte: El Medio Natural.

Infantes V.J. 1962. Estudio taxonómico, histológico y etnobotánico de algunas plantas útiles del Perú. Contribución a la Etnobotánica Peruana. Revista de Ciencias. LXIV. N519-520. : 35-72.

López M.A. 1983. Especies vegetales comunes de la región norte del Perú. Boletín de la Sociedad Botánica de La Libertad. Vol.XII. N¹-2. : 1-29.

Mac Bride J.F. 1936-1972. Flora of Perú. Field Museum of Natural History. Botanical Series. Vol.XIII

Meza I. 1991. Etnobotánica de la Isla Alao. Archipiélago de Chiloe, Chile. Boletín Nacional de Historia Natural. $\mathrm{N}^{\circ} 4$. : 39-78.

Polia M.M. 1989. Las Lagunas de los Encantos. Medicinal tradicional andina del Perú septentrional. Segunda edición. CEPESER. Piura.

Soukup J. 1987. Vocabulario de los Nombres 
Vulgares de la Flora Peruana y Catálogo de los Géneros. Editorial Salesiana. Lima. Perú. 436 p.

Comunicación personal: Sr. Angel Córdova Guerrero,

Sr. Manuel Córdova Guerrero, Sr. Gerardo Peña y

Sr. Francisco Chumacero López.

\section{ANEXOS: TABLAS Y FIGURAS}

Tabla 1: Lista alfabética de los nombres vulgares y sus equivalentes latinos de especies utilizadas en Huaylingas y Santo Domingo. Cuenca La Gallega. Santo Domingo. Morropón. Piura.

\begin{tabular}{|c|c|c|}
\hline Nombre Común & Nombre Científico & Familia \\
\hline Achicoria & Hypochoeris sp. & Asteraceae \\
\hline Ajo & Allium sativum $\mathrm{L}$. & Liliaceae \\
\hline Alcanfor & Eucalyptus globullus Labillardiere & Myrtaceae \\
\hline Amor seco & Bidens pilosa $\mathrm{L}$. & Asteraceae \\
\hline Arrayán & Eugenia myrobalana DC. & Myrtaceae \\
\hline Arveja & Pisum sativum $\mathrm{L}$. & Fabaceae \\
\hline Bejuco colorado & $\begin{array}{l}\text { Muehlenbeckia hastulata (Sm.) } \\
\text { Jhons. }\end{array}$ & Polygonaceae \\
\hline Caña de Guayaquil & Bambusa guadua H.\& B. & Poaceae \\
\hline Cascarilla & Cinchona sp. & Rubiaceae \\
\hline Cebada & Hordeum vulgare $\mathrm{L}$. & Poaceae \\
\hline Cebolla & Allium cepa L. & Liliaceae \\
\hline Clarín & Monnina salicifolia R.\& P. & Polygalaceae \\
\hline Corazoncillo & Hypericum silenoides Jussieu & Clusiaceae \\
\hline Cucharillo & Oreocallis grandiflora (Lam.) Brown & Proteaceae \\
\hline Chachacomo & Escallonia resinosa (R \& P) Pers. & Grossulariaceae \\
\hline Chicope & Carica pubescens (A.DC.)Solm.-Laub. & Caricaceae \\
\hline Chilca & Baccharis latifolia (R.\& P.) Pers. & Asteraceae \\
\hline Chin-chin & Acnistus arborescens (L.) Schlech. & Solanaceae \\
\hline Chin-chin serrano & lochroma grandiflorum Bentham & Solanaceae \\
\hline Chinchahual & Hypericum laricifolium Jussieu & Clusiaceae \\
\hline Choclo del Inca & Dalea coerulea (L.f.) Schin.\& Thell. & Fabaceae \\
\hline Chogolo & Boehmeria aff. caudata Swartz & Urticaceae \\
\hline Churgún & Clusia sp. & Clusiaceae \\
\hline Frijol de vida & Phaseolus polyanthus Greenman & Fabaceae \\
\hline Hierba de la araña & Spermacoce tenuior $\mathrm{L}$. & Rubiaceae \\
\hline Hierba de la quemazón & Alonsoa meridionalis (L.f.) Kuntze & Scrophulariaceae \\
\hline Hierba de la rabia & Oenothera rosea Aiton & Onagraceae \\
\hline Hierba de la sarna & Salvia cf. oppositiflora R.\& P. & Lamiaceae \\
\hline Hierba del anís & Hypericum sp. & Hyppericaceae \\
\hline Hierba del chucaque & Nothoscordum cf. andicola Kunth & Liliaceae \\
\hline Hierba del huisco & Lycopersicon cf. peruvianum (L.) Mill. & Solanaceae \\
\hline Hierba del quinde & Salvia cf. rhombifolia R.\& P. & Lamiaceae \\
\hline Hierba dulce & Muehlenbeckia volcanica (Ben.) Endl. & Polygonaceae \\
\hline Hierba Julia & Hymenostephium sp. & Asteraceae \\
\hline Higuerón & Ficus sp. & Moraceae \\
\hline Huaco & $N N$ & Lauraceae \\
\hline Huacum & Baccharis grandicapitulata Hieronym. & Asteraceae \\
\hline Huaminga & Berberis lutea R.\& P. & Berberidaceae \\
\hline Huancuncillo & Diplostephium sp. & Asteraceae \\
\hline Illirque & Axinaea cf. oblongifolia (Cog.) Wur. & Melastomataceae \\
\hline Lanche & Myrcia sp. & Myrtaceae \\
\hline Lanche de Dios & $N N$ & Myrtaceae \\
\hline Llantén serrano & Satureja pulchella (H.B.K.) Briquet. & Lamiaceae \\
\hline Maíz & Zea mays $\mathrm{L}$. & Poaceae \\
\hline Manzanilla & Matricaria recutita L. & Asteraceae \\
\hline Mastrante & Lippia cf. alba (Miller) N.E.Brown & Verbenaceae \\
\hline Menta & NN & Lamiaceae \\
\hline Mollaca & $N N$ & NN \\
\hline Mosquero & Croton baillonianus Muell.Arg. & Euphorbiaceae \\
\hline Nogal & Juglans neotropica Diels & Juglandaceae \\
\hline Oca & Oxalis tuberosa Molina & Oxalidaceae \\
\hline Olluco & Ollucus tuberosus Caldas & Basellaceae \\
\hline Pajul & Erythrina edulis Triana ex Micheli & Fabaceae \\
\hline Palo blanco & Croton lechleri Muell. Arg. & Euphorbiaceae \\
\hline
\end{tabular}

\begin{tabular}{|c|c|c|}
\hline Paltón & Persea caerulea (R.\& P.) Mez & Lauraceae \\
\hline Рapa & Solanum tuberosum $\mathrm{L}$. & Solanaceae \\
\hline Perlilla & Galium hypocarpium (L.) End.ex Gris. & Rubiaceae \\
\hline Planta de mote & NN & NN \\
\hline Polen de vaca & Minthostachys sp. & Lamiaceae \\
\hline Poleo blanco & NN & NN \\
\hline $\begin{array}{l}\text { Poleo de madural } \\
\text { lúcuma }\end{array}$ & Minthostachys tomentosa (Bent.) Epl. & Lamiaceae \\
\hline Poleo grande & Otholobium mexicanum (L.f.) Grimes & Fabaceae \\
\hline Puchuguero & Ocotea cernua (Nees) Mez & Lauraceae \\
\hline Pulén de tomar & Otholobium pubescens (Poir.) Grimes & Fabaceae \\
\hline Pulén de vaca & $\begin{array}{l}\text { Otholobium holosericeum (Bar.) } \\
\text { Grim. }\end{array}$ & Fabaceae \\
\hline Putaya & Rumex conglomeratus Murray & Polygonaceae \\
\hline Quínua negra & Amaranthus sp. & Amaranthaceae \\
\hline Romerillo & NN & NN \\
\hline Ruda & Ruta graveolens $\mathrm{L}$. & Rutaceae \\
\hline Salvia real & Salvia corrugata M. Vahl. & Lamiaceae \\
\hline Salvia real chiquita & Lepichinia cf. meyeni (Walp.) Eplin. & Lamiaceae \\
\hline Santa María & Buddleja americana L. & Loganiaceae \\
\hline Sauco & Cestrum aff. auriculatum L'Héritier & Solanaceae \\
\hline Shimir & Mauria sp. & Anacardiaceae \\
\hline Shimir colorado & NN & NN \\
\hline Trencilla & Lycopodium thyoides Willd. & Lycopodiaceae \\
\hline Trigo & Triticum aestivum $\mathrm{L}$. & Poaceae \\
\hline Valeriana & NN & Ericaceaev \\
\hline Verbena & Verbena sp. & Verbenaceae \\
\hline Vira-vira & Achyrocline alata (H.B.K.) DC & Asteraceae \\
\hline Yacuguero & Vernonia ferruginea Lessing & Asteraceae \\
\hline Yuto & Myrsine oligophylla Zalbruckner & Myrsinaceae \\
\hline Yuye & Tournefortia sp. & Boraginaceae \\
\hline Zambumba & NN & Cucurbitaceae \\
\hline Zanahoria & Daucus carota L. & Apiaceae \\
\hline Zarcilleja chiquita & Brachyotum sp. & Melastomataceae \\
\hline
\end{tabular}

Tabla 2: Plantas útiles de Huaylingas y Santo Domingo, ordenadas según su categoría de uso. Cuenca La Gallega. Morropón. Piura.

\begin{tabular}{|c|c|c|}
\hline CATEGORIA & & PLANTAS \\
\hline ALIMENTICIIAS & Cereales & Cebada, Maíz, Trigo. \\
\hline BEBIDAS & Legumbres & Arveja, Pajul, Frijol de vida. \\
\hline \multirow[t]{6}{*}{ FRUTALES } & Tuberosa & Oca, Olluco, Papa. \\
\hline & Hortalizas & $\begin{array}{l}\text { Ajo, Cebolla, Zanahoria, } \\
\text { Zambumba. }\end{array}$ \\
\hline & Nueces & Nogal. \\
\hline & Sazonadoras & Romerillo. \\
\hline & Bebidas & $\begin{array}{l}\text { Culén, Huancuncillo, Lanche, } \\
\text { Lanche de Dios, Manzanilla, } \\
\text { Pulén de tomar. }\end{array}$ \\
\hline & Frutales & Arrayán, Chicope \\
\hline $\begin{array}{l}\text { DE CARPINTERIA Y } \\
\text { CONSTRUCCION }\end{array}$ & \multicolumn{2}{|c|}{$\begin{array}{l}\text { Alcanfor, Caña de Guayaquil, Chachacomo, Chilca, Chivato, } \\
\text { Huaco, Illirque, Nogal, Paltón, Puchuguero, Shimir }\end{array}$} \\
\hline DE HIGIENE & \multicolumn{2}{|l|}{ Cucharillo. } \\
\hline FORRAJERAS & \multicolumn{2}{|l|}{ Nudillo, Suro. } \\
\hline MEDICINALES & \multicolumn{2}{|c|}{$\begin{array}{l}\text { Achicoria, Alcanfor, Amor seco, Bejuco colorado, Cascarilla, } \\
\text { Clarín, Chin-chin, Chin-chin serrano, Chinchahual, Choclo del } \\
\text { Inca, Hierba de la araña, Hierba de la quemazón, Hierba de la } \\
\text { rabia, Hierba de la sarna, Hierba del anís, Hierba del } \\
\text { chucaque, Hierba del huisco, Hierba del quinde, Hierba dulce, } \\
\text { Hierba Julia, Huacum, Huaminga, Huancuncillo, Llantén } \\
\text { serrano, Manzanilla, Mastrante, Menta, Mollaca, Perlilla, } \\
\text { Planta de mote, Polen de vaca, Poleo blanco, Poleo de madu- } \\
\text { rar lúcuma, Poleo grande,Putaya, Quínua negra, Salvia real, } \\
\text { Salvia real chiquita, Santa María, Sauco, Shimir colorado, } \\
\text { Valeriana, Verbena, Vira-vira, Yuye, Zarcilleja chiquita. }\end{array}$} \\
\hline $\begin{array}{l}\text { PARA LA BUENA } \\
\text { SUERTE }\end{array}$ & \multicolumn{2}{|c|}{ Ruda, Trencilla. } \\
\hline PARA LEÑA & \multirow{2}{*}{\multicolumn{2}{|c|}{$\begin{array}{l}\text { Alcanfor, Arrayán, Chogolo, Churgún, Mosquero, Palo blanco, } \\
\text { Paltón, Yacuguero, Yuto. } \\
\text { Alcanfor, Higuerón. }\end{array}$}} \\
\hline PARA REFORESTAR & & \\
\hline
\end{tabular}


Tabla 3: Formas de uso de plantas colectadas en Huaylingas y Santo Domingo. Cuenca La Gallega. Santo Domingo. Morropón. Piura.

\begin{tabular}{|c|c|}
\hline NOMBRE COMUN & FORMA DE USO \\
\hline Achicoria & Para el paludismo \\
\hline Ajo & Como cultivo \\
\hline Alcanfór & Para los bronquios, resfrío y baños de vapor \\
\hline Amor seco & Para huesos rotos \\
\hline Arrayán & Para leña y como frutal \\
\hline Arveja & Como cultivo \\
\hline Bejuco colorado & Para regular la sangre y recaída después del parto \\
\hline Caña de Guayaquil & Para construcción \\
\hline Cascarilla & Para eliminar parásitos \\
\hline Cebada & Como cultivo \\
\hline Cebolla & Como cultivo \\
\hline Clarín & Para dolores (se frota con primera) \\
\hline Cucharillo & Para eliminar el sarro \\
\hline Culén & En infusión para tomar \\
\hline Chachacomo & Para madera \\
\hline Chicope & Como frutal \\
\hline Chilca & Como fibra para amarras de techos \\
\hline Chin-chin & Para el aire \\
\hline Chin-chin serrano & Para erisipela, hinchazón, heridas, granos y lavados \\
\hline Chinchahual & Para el paludismo \\
\hline Choclo del Inca & Para el susto \\
\hline Chogolo & Para leña \\
\hline Churgún & Para leña \\
\hline Frijol de vida & Las semillas para comer \\
\hline Hierba de la araña & Emética, como sustituto de la ipecacuana \\
\hline Hierba de la quemazón & Para quemaduras \\
\hline Hierba de la rabia & Para los nervios, riñones y eliminar bichos \\
\hline Hierba de la sarna & Para la sarna \\
\hline Hierba del anis & Para la vejiga \\
\hline Hierba del chucaque & Para el susto y eliminar parásitos \\
\hline Hierba del huisco & Para frotaciones, el aire y dolor de barriga \\
\hline Hierba del quinde & Medicinal \\
\hline Hierba dulce & La raíz para hemorrágeas en la mujer y descensos \\
\hline Hierba Julia & Para reponerse del embarazo \\
\hline Higuerón & Para reforestar \\
\hline Huaco & En carpintería \\
\hline Huacum & Para resfrío y reumatismo \\
\hline Huaminga & Cocinado para mal de espaldas y malestar \\
\hline Huancuncillo & Se toma con salvia real y otras hierbas \\
\hline Illirque & Para hacer vigas \\
\hline Lanche & En infusión para tomar \\
\hline Lanche de Dios & En infusión para tomar \\
\hline LLantén serrano & Para el resfrío \\
\hline Maíz & Como cultivo \\
\hline Manzanilla & En infusión para el dolor de barriga, como agua de tiempo y \\
\hline Mastrante & Como purgante y agua de tiempo. \\
\hline Menta & Para el resfrí y dolor de estómago \\
\hline Mollaca & La flor y el fruto para hemorrágeas en la mujer \\
\hline Mosquero & Para leña \\
\hline Nogal & En carpintería \\
\hline Oca & Como cultivo \\
\hline Olluco & Como cultivo \\
\hline Pajul & Las semillas para comer \\
\hline Palo blanco & Para leña \\
\hline Paltón & En carpintería y para leña \\
\hline Papa & Como cultivo \\
\hline Perlilla & En infusión para tomar y en frotaciones para la viruela \\
\hline Planta de mote & Para el malestar \\
\hline Polen de vaca & Para los riñones \\
\hline Poleo blanco & Mezclado con otras hierbas \\
\hline Poleo de madural lúcuma & Para el costipado y la gripe \\
\hline Poleo grande & En infusión para tomar y el resfrío \\
\hline Puchuguero & En carpintería \\
\hline Pulén de tomar & Como agua de tiempo \\
\hline Putaya & Las hojas para los granos y la raíz para los oídos \\
\hline Quinua negra & Para la circulación de la sangre y como defensivo \\
\hline Romerillo & Como condimento para cocinar \\
\hline Ruda & Para el aire y la suerte \\
\hline Salvia real & Para el resfrío \\
\hline Salvia real chiquita & Para resfrío y dolor de estómago \\
\hline Santa María & Para frotaciones, dolor de cabeza y el aire \\
\hline Sauco & Para frotaciones \\
\hline Shimir & Para madera \\
\hline Shimir colorado & Caldeado para mordedura de perro \\
\hline Trencilla & Para la suerte \\
\hline Trigo & Como cultivo \\
\hline Valeriana & Para descensos en la mujer y malestar en general \\
\hline Verbena & Para el chucaque \\
\hline Vira-vira & Para lavar heridas \\
\hline Yacuguero & Para leña \\
\hline Yuto & Para leña \\
\hline Yuye & Para picaduras de araña \\
\hline Zambumba & Como cultivo \\
\hline Zanahoria & Como cultivo \\
\hline Zarcilleja chiquita & Para hemorrágeas \\
\hline
\end{tabular}

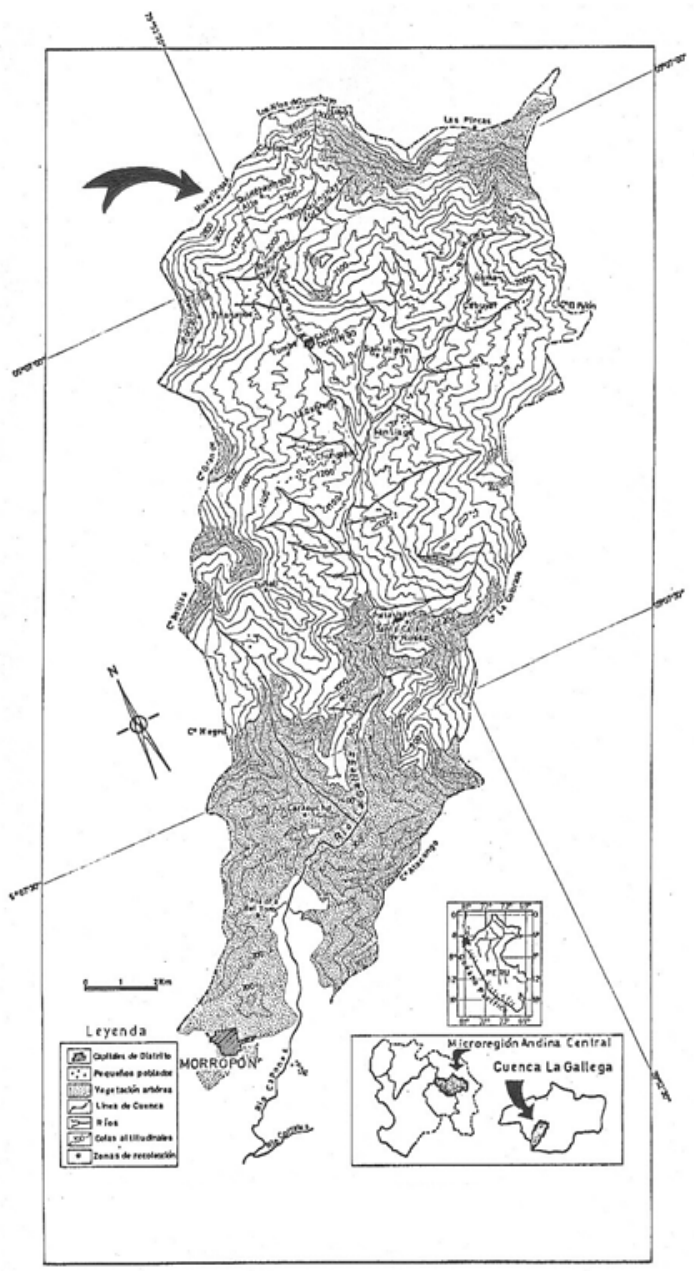

Figura 1: Ubicación del poblado de Huaylingas en la Cuenca la Gallega Santo Domingo. Morropón. Piura 


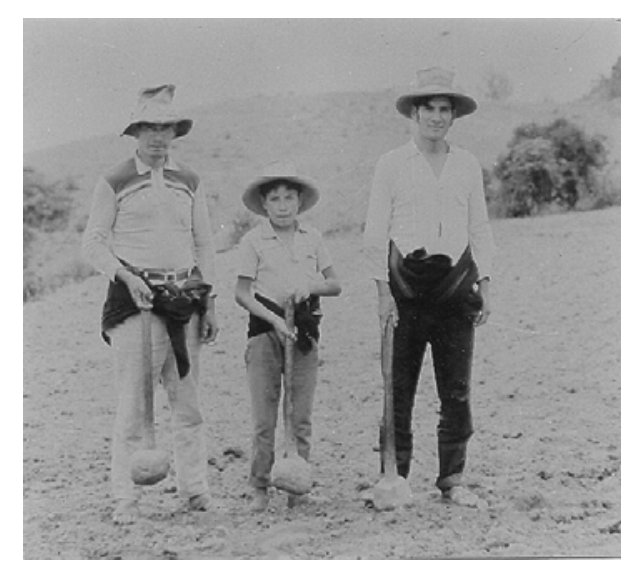

Foto 1. El pilón, herramienta hecha de madera para romper terrones durante la preparación del campo de cultivo. (Tomada en Quinchado Grande a 3000 m.s.n.m.)

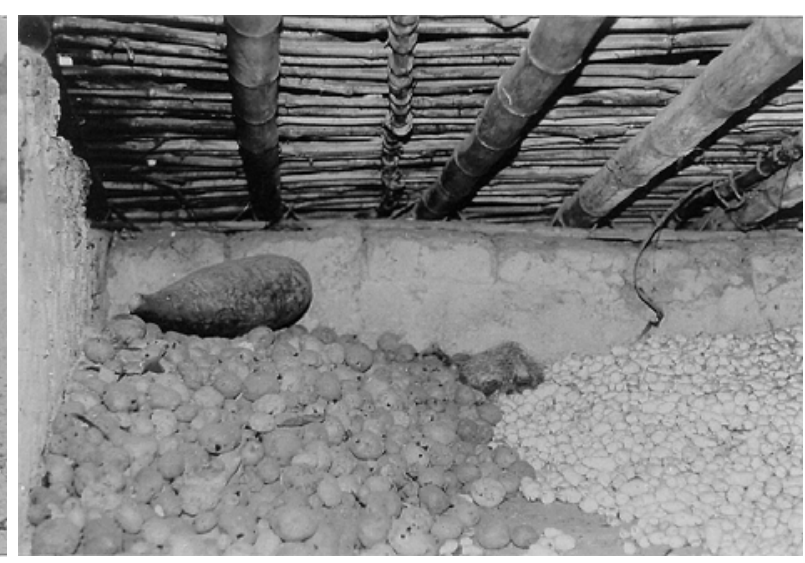

Foto 2. El soberado o tabanque, técnica de conservación de tuberosas. (Tomada en Florecer a 3200 m.s.n.m.)

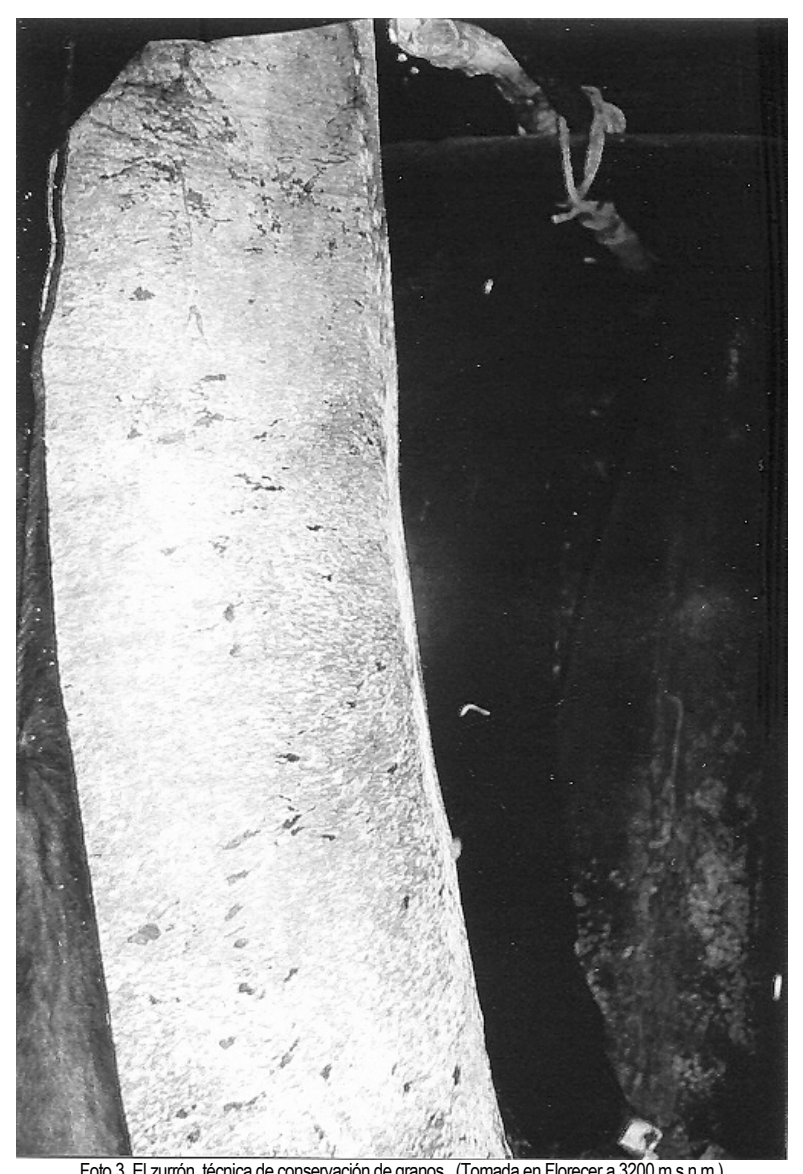

Foto 3. El zurrón, técnica de conservación de granos. (Tomada en Florecer a 3200 m.s.n.m.) 